\title{
A case of meningitis caused by Streptococcus pyogenes in a previously healthy woman
}

\author{
Mehmet Ulug ${ }^{1}$, Nuray Can Ulug ${ }^{2}$, Mustafa Kemal Celen ${ }^{3}$, Mehmet Faruk Geyik ${ }^{3}$, Celal Ayaz ${ }^{3}$ \\ ${ }^{1}$ Midyat State Hospital, Department of Infectious Diseases and Clinic Microbiology \\ ${ }^{2}$ Midyat State Hospital, Department of Neurology \\ ${ }^{3}$ Dicle University Medical School, Department of Infectious Diseases and Clinic Microbiology
}

\begin{abstract}
Background: Streptococcus pyogenes is a well-known cause of a variety of clinical infections including local symptoms such as tonsillopharyngitis, cervical lymphadenitis, otitis media, cellulites, erysipelas, as well as more severe diseases such as scarlet fever, osteomyelitis, necrotizing fasciitis, sepsis, and toxic shock syndrome. However, acute bacterial meningitis caused by this pathogen is unusual.

Objective: We report a case of group A streptococcus (GAS) meningitis in a previously healthy woman with a dramatically rapid course and fatal outcome.

Case: A 41-year-old previously healthy woman presented a history of fever, headache, vomiting, and sore throat of three days' duration. Neurological examination revealed diminished consciousness and neck rigidity. The cerebrospinal fluid (CSF) was turbid with10,000 leukocytes $/ \mathrm{mm}^{3}$. Direct examination of CSF showed Gram-positive cocci in chains, and cultures yielded S. pyogenes. Blood cultures yielded growth of $S$. pyogenes. The patient was treated initially with ceftriaxone ( $4 \mathrm{~g} /$ day) and the control CSF examination was not changed on the third day, so vancomycin ( $2 \mathrm{~g} / \mathrm{day})$ was added to the treatment; however, she died on the fourth day of the treatment.

Conclusion: S. pyogenes meningitis is uncommon and the incidence seems to be persistently low; nevertheless, clinicians should be aware that sporadic cases may occur and may have a fulminant course with a relevant neurological sequel.
\end{abstract}

Key words: Streptococcus pyogenes, meningitis, mortality, Turkey

J Infect Developing Countries 2009; 3(3):241-244.

Received 27 - October 2008 Accepted 16 February 2009

Copyright (c) 2009 Ulug et al. This is an open-access article distributed under the Creative Commons Attribution License, which permits unrestricted use, distribution, and reproduction in any medium, provided the original work is properly cited.

\section{Introduction}

Streptococcus pyogenes, group A streptococcus (GAS), is a major human bacterial pathogen that frequently colonizes in the throat. Pharyngeal carriage rates among normal schoolchildren vary with geographic location and season of the year. Carriage rates of $15-20 \%$ in children have been noted in several studies; however, they are considerably lower in adults [1].

This ubiquitous organism is the most frequent bacterial cause of acute pharyngitis, and it also gives rise to a variety of coetaneous and systemic infections. Reports of GAS invasive disease have increased worldwide in recent years and it has reemerged as a public health threat [2]. Despite this increase, GAS bacterial meningitis remains uncommon and accounts for less than $0.2 \%$ of all cases of bacterial meningitis $[3,4]$. Persons of all ages can be infected and although some patients also have predisposed underlying illnesses, most are healthy
[5]. GAS frequently colonizes the oropharynx; however, it does not usually invade the central nervous system (CNS) directly and the pathogenesis of primary GAS meningitis remains unclear. In more recent years, GAS meningitis has been associated with a lower mortality rate if treated promptly [6]. We report a case of GAS meningitis in a previously healthy woman with a fulminant course and fatal outcome. To the best of our knowledge, this is the first case report of meningitis caused by $S$. pyogenes in an adult from Turkey.

Case

In February 2006, a previously healthy 41-yearold woman was admitted to the University of Dicle Hospital in Diyarbakir, Turkey. She complained of fever, headache, vomiting and sore throat of eight days' duration. A week prior to admission, she reported to a community hospital emergency department with a one-day history of fever and 
repetitive vomiting. She was diagnosed with acute pharyngitis and prescribed oral azithromycin and acetaminophen at home but had no relief from her symptoms. Apart from her diagnosis of acute pharyngitis, the patient's medical history was normal. She had no previous history of ear discharge or any cranial surgery, and no family history suggesting any significant illness, including tuberculosis.

On the day of admission, she was unconscious and her temperature was $38.5^{\circ} \mathrm{C}$. She had poor general appearance. Blood pressure, pulse and respiratory rate were $110 / 60 \mathrm{mmHg}, 96 /$ minute, and 48/minute, respectively. The respiratory system, cardiovascular system, and abdomen were all normal. Neurological examination revealed neck rigidity. Because of her examination and history, bacterial meningitis was suspected and a diagnostic lumbar puncture (LP) was performed. She was rapidly transferred to the intensive care unit because of her acute deterioration at our institution. In the intensive care unit, endotracheal intubation was performed and she was stabilized with mechanical ventilation. Her Glasgow Coma Scale Score was 5, and she exhibited decerebrate posturing due to painful stimuli. Pupils were equal but weakly reactive to light with absent oculocephalic reflexes. No skin rashes were detectable.

Laboratory investigations revealed a hemoglobin count of $8.3 \mathrm{~g} / \mathrm{dl}$ and a total leukocyte count of $22,700 / \mathrm{mm}^{3}$ (polymorphs $94 \%$ ). The $\mathrm{C}$ reactive protein was elevated up to $351 \mathrm{mg} / \mathrm{dl}$ and ESR was $57 \mathrm{~mm} / \mathrm{h}$. Serum electrolytes, and renal and liver function tests were within normal limits. The cerebrospinal fluid (CSF) was turbid with 10,000 leucocytes $/ \mathrm{mm} 3$ (95\% polymorphs and 5\% lymphocytes). The CSF sugar was within normal limits $(61 \mathrm{mg}$, range $50-80 \mathrm{mg} / 100 \mathrm{ml}$ ) and proteins were $474 \mathrm{mg} / 100 \mathrm{ml}$. Direct examination of CSF showed Gram-positive cocci and chains. The peripheral blood smear was negative for malaria parasite. Serum C3, C4, complement function and immunoglobulin concentrations were normal, and urinalysis revealed no evidence of infection. CSF, blood, urine, and throat cultures had been performed before the treatment was started.

She was initially treated with ceftriaxone (4g/day). The CSF culture subsequently grew $S$. pyogenes that were sensitive to penicillin, ceftriaxone and vancomycin. The initial blood culture obtained prior to antibiotic therapy was also positive for GAS, identified by an antigen-agglutination test as group A streptococci. A chest radiograph was normal, and an emergent unenhanced CT scan of the brain showed cerebral edema and left-sided mastoiditis as a possible cause of meningitis.

Given the clinical picture consistent with increased intracranial pressure, the patient was mechanically hyperventilated and received a trial of mannitol therapy with no benefit. Control lumber puncture was done on the third day of treatment, but there was no change in CSF examination. Vancomycin (2g/day) was therefore added to the treatment. Three days after admission, her condition deteriorated further in spite of supportive treatment, and she died on the fourth day of admission. Throat cultures from her close family contacts were negative. Her GAS isolates were not available for phage typing, and an autopsy was refused.

\section{Discussion}

GAS meningitis is uncommon and is described in several reports as comprising less than $0.2 \%$ to $0.6 \%$ of all cases of meningitis [9]. Unlike Haemophilus influenzae and, Streptococcus pneumoniae in which meningitis is considered to be secondary to bacteraemia, and also unlike Neisseriae meningitis in which meningitis may be secondary to invasion of oropharyngeal mucosa and direct invasion of the CSF, the pathogenesis of GAS meningitis has not been entirely explained [9]. Generally, it is not present as a complication of the invasive disease and there is a frequent association with contagious infectious foci of the upper respiratory tract. Furthermore, some risk factors have been described, including neurosurgery, skull fractures, CSF leaks, infection, colonization of the upper respiratory tract, skin lesions. However, GAS meningitis also occurs in healthy individuals who do not have predisposed conditions and who do not present clear evidence of a contiguous focus [10]. In cases reviewed by Chow and Muder [11], the most common associated condition was otitis media. In studies with children, Shetty et al. [5] documented 30 cases of GAS meningitis over a 25-year period from 1976 to 2001. Among these, $52 \%$ had a primary focus of infection in the ear, nose, and throat area. The history of the patient whom we have described had pharyngitis a week prior to the onset of meningitis which,, along with the mastoiditis assigned from the CT scan, may have been the source of infection. Shetty et al. [5] showed that blood culture grew GAS in $59 \%$ of cases and in our case blood cultures yielded growth of $S$. pyogenes as well. In the Netherlands, a case series of 41 patients aged 16 years and older with GAS 
meningitis was published in 2002 [4]. In this report, the mortality rate was $27 \%$, a statistic that contrasts with data from the literature that describes a mortality rate of $5-10 \%[3,6,11]$. Our patient had a similar fulminant course and died.

The clinical presentation of GAS meningitis is not different from the other bacterial meningitis [10]. The CSF biochemical and cytological profiles are usually consistent with purulent meningitis, with neutrophilic leukocytosis in a majority of reported cases and a very low glucose level in about one-third of cases [12]. Similarly, the clinical course was associated with major complications including seizures (most frequently), coma, brain abscess, shock, syndrome of inappropriate antidiuretic hormone secretion, acute glomerulonephritis and renal failure, disseminated intravascular coagulation, and hepatitis [5]. However, neurological sequel in $36 \%$ of the survivors was documented [4].

In the present study, specimens of CSF were cultured on sheep blood (5\%), chocolate, and MacConkey agar plates. The plates were incubated at $35^{\circ} \mathrm{C}$ aerobically (MacConkey agar), under 5\% carbon dioxide (blood and chocolate agar), and examined at 18 to 24 hours. Isolates showing betahemolysis were smeared and Gram stained. Those that were typical of streptococci in appearance were grouped by a battery of tests, including bacitracin and trimethoprim-sulphamethoxazol sensitivity, sodium hippurate hydrolysis, bile tolerance, and hydrolysis of esculin. Plates that showed heavy beta-hemolysis within the zone of inhibition of normal flora surrounding the SXT (1.25 mg trimethoprim and $23.75 \mathrm{mg}$ sulphamethoxazol) disk (Oxoid) and the inhibition of growth around the 0.04-unit bacitracin (BBL Microbiological Systems; Taxo A) disk were interpreted as positive for group A beta-hemolytic streptococci. Group B streptococci were identified by the hydrolysis of sodium hippurate, and group D streptococci were identified by the ability of the organism to hydrolyze esculin in the presence of $40 \%$ bile [12]. Serological typing was used for strains that could not be grouped by the above matrix of tests as well as for confirmation of group B or group A identification (Phadebact Streptococcus Tests; Pharmacia Diagnostics).

Despite the emergence of resistance to several antibiotics, GAS is still sensitive to most antibiotics, with penicillin being the first choice of treatment [2]. Third-generation cephalosporin, especially ceftriaxone, were suitable alternative agents [8]. Our case was initially treated with ceftriaxone, which constitutes the recommended empirical treatment of meningitis cases in our environment. In reviewing the literature, we also observed that the initial empirical treatment constitutes the use of third-generation cephalosporin. In some cases, even after the etiological diagnosis, the antibiotic treatment was not changed to penicillin despite $S$. pyogenes having a preserved sensitivity to penicillin [10]. In our case, $S$. pyogenes was susceptible to all antibiotics except gentamicin. Once CSF infection is proven, antibiotics must be adjusted according to the microorganism identified but the duration of therapy can be variable because there is currently no consensus in the literature [6].

In conclusion, GAS meningitis remains an unusual manifestation of invasive GAS disease in adults. It usually occurs in association with some other focus of infections such as otitis media, mastoiditis, and pharyngitis. Clinicians should be aware that sporadic cases may occur and may have a fulminant course with relevant neurological sequel. If the diagnosis is made early, the outcome tends to be favorable.

\section{References}

1. Bisno AL, Stevens DL. Streptococcus pyogenes (2005) In: Mandell GL, Bennet GE, Dolin R eds. Principles and Practice of Infectious Diseases. $6^{\text {th }}$ ed. Philadelphia: Churchill Livingstone. 2362-2379.

2. Mani R, Mahadevan A, Pradhan S, et al. (2007) Fatal group A streptococcal meningitis in an adult. Indian $\mathrm{J}$ Med Microbiol 25: 169-170.

3. Mathur P, Arora NK, Kapit A, Das BK (2004) Streptococcus pyogenes meningitis. Indian J Pediatr; 71: 423-426.

4. van de Beek D, DE Gans J, Spanjaard L, Sela S, Vermeulen M, Dankert J (2002) Group A streptococcal meningitis in adults: report of 41 cases and review of the literature. Clin Infect Dis 34: 32-36.

5. Shetty AK, Frankel LR, Maldanado Y, Falco DA, Lewis DB (2001) Group A streptococcal meningitis: Report of a case and review of literature since 1976. Pediatr Emerg Care 17: 430-434.

6. Pettersen G, Ovetchkine P, Tapiero B (2005) Group A streptococcal meningitis in a pediatric patient following cochlear implantation: Report of the first case and review of the literature. J Clin Microbiol 43: 5816-5818.

7. Moses A, Beeri M, Engelhard D (1998) Group A streptococcal meningitis: Report of two cases. J Infect 36: 116-118.

8. Arnoni MV, Berezin EN, Safadi MAP, Almeida FJ, Lopes CRC (2007) Streptococcus pyogenes meningitis in children: Report of two cases and literature review. Braz J Infect Dis 11: 375-377.

9. Chow JW, Muder RR (1992) Group A streptococcal meningitis. Clin Inf Dis 14: 418-421.

10. Huang SC (1982) Bacterial meningitis: a pediatrician's unusual encounters. J Fla Med Assoc 69: 1002-1003. 
11. Perara N, Abulhoul L, Green MR, Swann RA (2005) Group A streptococcal meningitis: case report and review of the literature. J Infect 51: e1-e4.

12. Altindis M, Aktepe OC, Kocagoz T (2004) Comparison of Dio-Bacit, bacitracin-trimethoprim/sulphametoxazole and latex agglutination in the diagnosis of group A betahemolytic streptococci. Yonsei Med J 45: 56-60.

\section{Corresponding Author}

Mehmet ULUĞ, Midyat Devlet Hastanesi, Enfeksiyon Hastalıkları ve Klinik Mikrobiyoloji Kliniği, 47100 Mardin, Turkey

Mobil phone: +905324475756, fax: +904826424539

Email: mehmetulug21@yahoo.com

Conflict of interest: No conflict of interest is declared. 